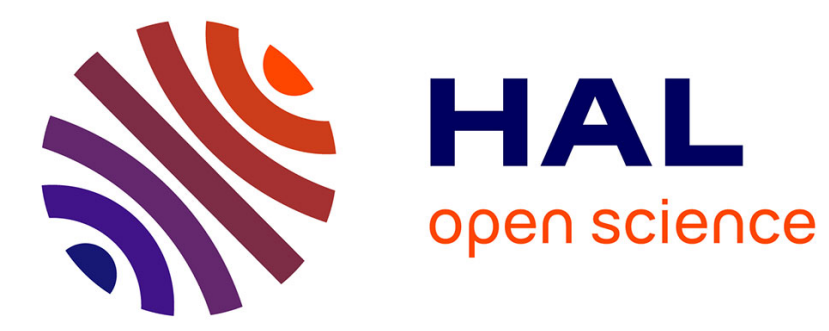

\title{
A discrete geometry approach for dominant point detection
}

Thanh Phuong Nguyen, Isabelle Debled-Rennesson

\section{To cite this version:}

Thanh Phuong Nguyen, Isabelle Debled-Rennesson. A discrete geometry approach for dominant point detection. Pattern Recognition, 2011, pp.32-44. inria-00526714

\section{HAL Id: inria-00526714 \\ https://hal.inria.fr/inria-00526714}

Submitted on 10 Dec 2010

HAL is a multi-disciplinary open access archive for the deposit and dissemination of scientific research documents, whether they are published or not. The documents may come from teaching and research institutions in France or abroad, or from public or private research centers.
L'archive ouverte pluridisciplinaire HAL, est destinée au dépôt et à la diffusion de documents scientifiques de niveau recherche, publiés ou non, émanant des établissements d'enseignement et de recherche français ou étrangers, des laboratoires publics ou privés. 


\title{
A discrete geometry approach for dominant point detection
}

Thanh Phuong Nguyen ${ }^{\mathrm{c}, * *}$, Isabelle Debled-Rennesson ${ }^{\mathrm{d}}$

\author{
${ }^{a}$ LORIA Nancy, Campus Scientifique - BP 239 \\ 54506 Vandouvre-lès-Nancy Cedex, France \\ ${ }^{b}$ LORIA Nancy, Campus Scientifique - BP 239 \\ 54506 Vandouvre-lès-Nancy Cedex, France
}

\footnotetext{
This work is supported by the ANR in the framework of the GEODIB project, BLAN06-2 134999.

Bthed on "Fast and robust dominant points detection on digital curves" by T.P. Nguyen and I. Debled-Rennesson in proceedings of ICIP'09 [1].

${ }^{*}$ Corresponding author

Email addresses: nguyentp@loria.fr (Thanh Phuong Nguyen), debled@loria.fr (Isabelle Debled-Rennesson)
} 


\title{
A discrete geometry approach for dominant point detection
}

\author{
Thanh Phuong Nguyen ${ }^{\mathrm{c}, * *}$, Isabelle Debled-Rennesson ${ }^{\mathrm{d}}$ \\ ${ }^{c}$ LORIA Nancy, Campus Scientifique - BP 239 \\ 54506 Vandouvre-lès-Nancy Cedex, France \\ ${ }^{d}$ LORIA Nancy, Campus Scientifique - BP 239 \\ 54506 Vandouvre-lès-Nancy Cedex, France
}

\begin{abstract}
We propose two fast methods for dominant point detection and polygonal representation of noisy and possibly disconnected curves based on a study of the decomposition of the curve into the sequence of maximal blurred segments [2]. Starting from results of discrete geometry [3,4], the notion of maximal blurred segment of width $\nu[2]$ has been proposed, well adapted to possibly noisy curves. The first method uses a fixed parameter that is the width of considered maximal blurred segments. The second method is deduced from the first one based on a multi-width approach to obtain a non-parametric method that uses no threshold for working with noisy curves. Comparisons with other methods in the literature prove the efficiency of our approach. Thanks to a recent result [5] concerning the construction of the sequence of maximal blurred segments, the complexity of the proposed methods is $O(n \log n)$. An application of vectorization is also given in this paper.
\end{abstract}

Key words: dominant point, corner detection, polygonal approximation

\section{Introduction}

The work on the detection of dominant points started from the research of Attneave [6] who proposed that the local maximum curvature points on a curve have a rich information content and are sufficient to characterize this curve. A method for detection of dominant points can lead to a good representation of a planar shape at different resolutions. In addition, a representation of a planar shape based on dominant points has some advantages. Firstly, it enables a high data reduction. Secondly, this representation concentrates on principal features of the shape, so it is efficient for shape matching, feature extraction or decomposition of the curve into meaningful parts. Therefore, these points have a critical role in curve approximation, shape matching and image matching [7]. They also lead to some applications in other domains of machine vision such as vector data compression [8]. Starting from Attneave's work, there are many existing methods for dominant points detection. Concerning this problem, several problems in this topic have been identified: evaluation, number of parameters, selection of starting point, multiscale, working with noisy curves, ...

This work is supported by the ANR in the framework of the GEODIB project, BLAN06-2 134999.

Based on "Fast and robust dominant points detection on digital curves" by T.P. Nguyen and I. Debled-Rennesson in proceedings of ICIP'09 [1].

${ }^{* *}$ Corresponding author

Email addresses: nguyentp@loria.fr (Thanh Phuong

Nguyen), debled@loria.fr (Isabelle Debled-Rennesson)
In general, we can classify these methods into 2 groups. The first one contains direct methods that determine dominant points such as high curvature value points by using curvature-based significant measures [9], or using alternative significant measures such as k-cosine [10], region of support (ROS) [11]. Rosenfeld and Johnston [10] used cosine of the angle between the arcs of length $\mathrm{k}$ on each side of a point (termed k-cosine) as curvature-based significant measure. Teh and Chin [11] proposed a non-parametric method for detecting dominant points. They used ROS as significant measure that is determined at each point $p_{i}$ thanks to its local properties (chord $p_{i-k} p_{i+k}$ and perpendicular distance from $p_{i}$ to this chord). The dominant points are finally detected by a non maximum suppression process. They also concluded in this work that the performance of a dominant point detection algorithm depends not only on the accuracy of significant measure, but also on the precise determination of ROS. Marji and Siy [12] determined ROS by using integral square error. They selected end points of support arm as dominant points upon the frequency of their selection. Other algorithms exploit the rule, iteration on neighbouring pixels. Sarkar [13] proposed a simple non-parametric method for detecting dominant points from a chained-code boundary. This algorithm examines the differential chain-code of the boundary points sequentially and confirms the significant points based on pure chain code manipulation. Cronin [14] assigned a special code to each boundary point based on the Freeman chain code [15] applied for its two immediate neighbours. The initial dominant points are the set 
of points with non-zero differential chain code. An elimination process is followed where the boundary is searched exhaustively for predefined sequences to eliminate them from initial dominant set.

The indirect methods are often based on polygonal approximation of the curve, the dominant points are deduced after doing this step. In these methods, the dominant points are detected as the vertex of approximated polygons. In addition, we can divide polygonal approximation methods into 3 principal approaches: sequential approach, split and merge approach, heuristic search one. For sequential approach, Ray and Ray [16] determined the longest possible line segments with minimum error. Kolesnikov [17] proposed a sub-optimal algorithm for polygon approximation of closed curves based on the corresponding optimal dynamic programming algorithm for open curves. Aoyama [18] used a linear scan to evaluate error conditions, if the conditions are not satisfied, a new segment search is started. The problem of this method is that sometimes the nodes do not correspond to the corners because a new vector is defined only when the conditions are violated. For split-and-merge methods [19, 20], lines are fitted to an initial segmentation of the boundary and then the least square error is computed. These methods then iteratively split a line if the error is too large and merge two points if the error is too small. Heuristic approach is used to reduce the complexity of an optimal algorithm for approximating polygon. Genetic algorithm [21], tabu search [22], ant colony search [23], fuzzy reasoning [24] are some popular techniques in this approach. Among 3 approaches, the sequential approach is simple and fast, but the quality of its result depends on the starting point. Recently, Masood [25] has proposed an efficient method that doesn't belong to any of the above groups. It is based on break point extraction. A break point is a point of which the chain code is different from that of the previous point. Break points are taken as initial set of dominant points. Each break point $D P_{j}$ is labeled by corresponding AVE(Associated Value Error) that is maximum perpendicular distance of all points between $D P_{j-1}$ and $D P_{j+1}$ from the straight line $D P_{j-1} D P_{j+1}$. An iterative process is applied to remove the break point which has the smallest AVE until the error approximation passes a threshold. This idea is similar to Latecki's approach [26].

Many methods for dominant point detection and polygonal approximation have been proposed. So, an evaluation measure for comparing these existing methods is also a critical request. Sarkar [13] proposed a trade off between the approximating error and the profit of high data reduction that are obtained by the method. The term compression rate $(C R)$ is used for determining the capacity of data reduction. So, he introduced the term figure of merit $(F O M)$ which is a division between $C R$ and ISE (integral square error) as an evaluation criterion for comparing dominant point detectors. Rosin [27] investigated evaluation measures which can be used to compare polygonal approximations with different number of line segments. The assessment is splitted into 2 components: fidelity and efficiency. The first component measures how well the method fits the curve relative to the optimal polygon concerning approximation error with the same number of line segments. The second one measures how compact the method represents the curve relative to the optimal polygon with the same error. A combined measure (Merit) is defined as the product of both components.

In this paper, we present a new and fast sequential method issued from theoretical results of discrete geometry, it only requires one parameter. It relies on the geometrical structure of the studied curve obtained by considering the decomposition of the curve into maximal blurred segments for a given width [2]. Thanks to this decomposition, a scan process for determining common zones of maximal blurred segments that contain dominant point candidates can be done efficiently. For each common zone, the corresponding dominant point is detected as its central point. Thanks to the advantage of blurred segment notion, our method can work naturally in different scales. In addition, it works naturally with possibly noisy or disconnected curves. These are good properties for shape matching of planar curves based on dominant points.

The rest of the paper is presented as follows. In section 2 , we recall theoretical results of discrete geometry used in this paper to analyze a curve. Section 3 describes our method for dominant point detection with a fixed parameter. Sections 4 introduces several experimental results and conclusions.

\section{Segmentation of a curve into maximal blurred segments}

\subsection{Discrete line and blurred segment}

We recall below several notions concerning discrete lines [28], blurred segments [4], and maximal blurred segments [2] which are used in the next section of this paper.

Definition 1. A discrete line $\mathcal{D}(a, b, \mu, \omega)$, with a main vector $(b, a)$, a lower bound $\mu$, and an arithmetic thickness $\omega$ (with $a, b, \mu$ and $\omega$ being integer such that the great common division of them is $1, \operatorname{gcd}(a, b)=1)$ is the set of integer points $(x, y)$ verifying $\mu \leq a x-b y<\mu+\omega$. Such a line is denoted by $\mathcal{D}(a, b, \mu, \omega)$.

For a simplified writing, Debled supposed in [4] that $0<$ $a<b$.

Let us consider $\mathcal{S}_{f}$ as a sequence of integer points.

Definition 2. A discrete line $\mathcal{D}(a, b, \mu, \omega)$ is said bounding for $\mathcal{S}_{f}$ if all points of $\mathcal{S}_{f}$ belong to $\mathcal{D}$.

Definition 3. A bounding discrete line $\mathcal{D}(a, b, \mu, \omega)$ of $\mathcal{S}_{f}$ is said optimal if its vertical distance (i.e. $\left.\frac{\omega-1}{\max (|a|,|b|)}\right)$ is minimal, i.e. if its vertical distance is equal to the vertical distance of the convex hull of $\mathcal{S}_{f}$. The vertical (resp. horizontal) distance of $\mathcal{D}(a, b, \mu, \omega)$ is equal to $\frac{\omega-1}{b}$ (resp. $\left.\frac{\omega-1}{a}\right)$. 


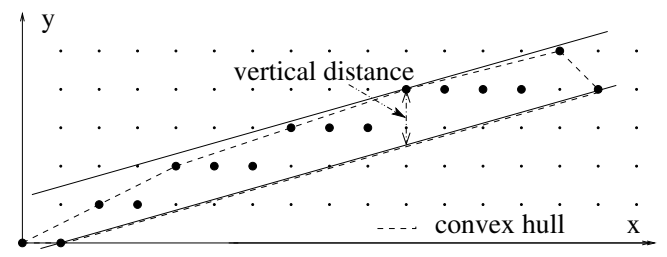

Figure 1: $\mathcal{D}(2,7,-8,11)$, the optimal bounding line of the set of points (vertical distance $=\frac{10}{7}=1.42$ ).

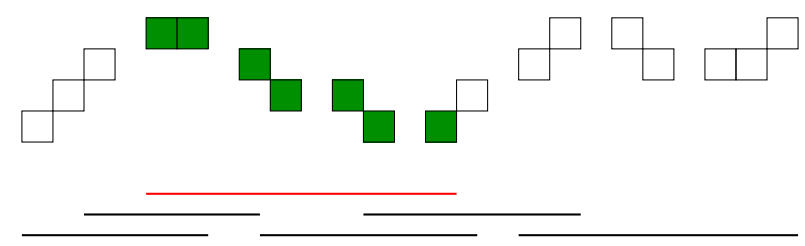

Figure 2: A maximal blurred segment of width 1 (in gray pixel) and the decomposition of the curve into the sequence of maximal blurred segments of width 1 (in lower part of figure).

This definition is illustrated in figure 1 and leads to the definition of the blurred segments.

Definition 4. A set $S_{f}$ is a blurred segment of width $\nu$ if its optimal bounding line has a vertical distance less than or equal to $\nu$ i.e. if $\frac{\omega-1}{\max (|a|,|b|)} \leq \nu$

Nguyen and Debled also introduced the notion of maximal blurred segment [2]. Let $\mathcal{C}$ be a discrete curve and $C_{i, j}$ a sequence of points of $\mathcal{C}$ indexing from i to $\mathrm{j}$. Let us suppose that the predicate " $C_{i, j}$ is a blurred segment of width $\nu$ " is denoted by $B S(i, j, \nu)$.

Definition 5. $C_{i, j}$ is called a maximal blurred segment of width $\nu$ and noted $M B S(i, j, \nu)$ iff $B S(i, j, \nu), \neg B S(i, j+$ $1, \nu)$ and $\neg B S(i-1, j, \nu)$ (see figure 2$)$.

\subsection{Construction of the sequence of maximal blurred seg- ments}

An algorithm is proposed in [2] to determine the sequence of maximal blurred segments of width $\nu$ of a discrete curve $C$ of $n$ points. The main idea is to maintain a blurred segment when a point is added (or removed) to (from) this one. It is based on 2 results: the dynamic maintenance of a convex hull in $O\left(\log ^{2} n\right)$ time proposed by Overmars et al. [29], the determination of height and width of a convex hull in $O\left(\log ^{2} n\right)$ time proposed by Buzer [30]. Thanks to this maintenance, the sequence of maximal blurred segment is incrementally constructed. The next maximal blurred segment is determined from the current one by using these rules below (see also algorithm 1).

- Insert a point at the end of the blurred segment while its width is less than $\nu$

- Remove a point at the beginning of the blurred segment while its width is greater than $\nu$

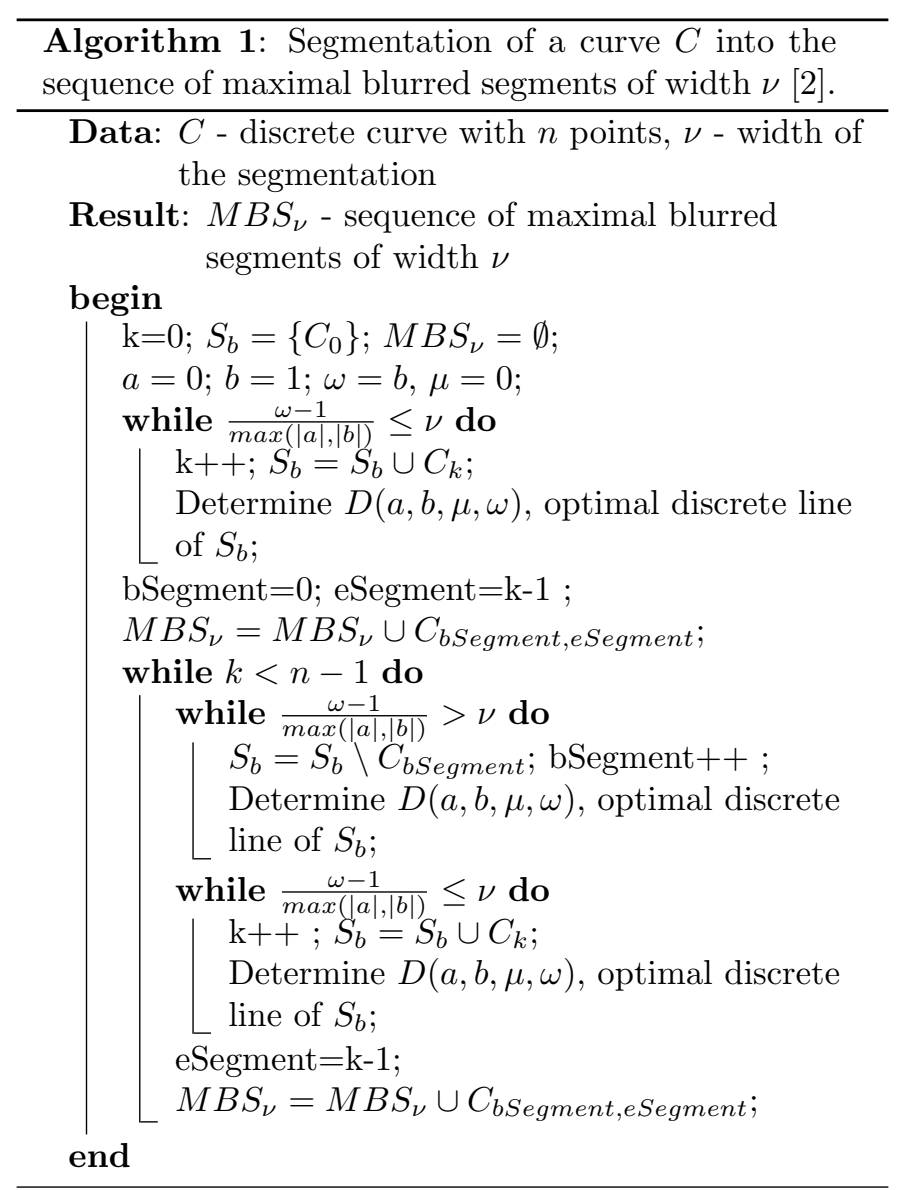

Let $C=\left\{C_{i}\right\}_{i=1 . . n}$ be a discrete curve and $M B S_{\nu}(C)$ the sequence of all maximal blurred segments of $\mathrm{C}$, in which the $i^{\text {th }}$ maximal blurred segment $M B S\left(B_{i}, E_{i}, \nu\right)$, is a set of points indexing from $B_{i}$ to $E_{i}$. Concerning the representation based on the sequence of maximal blurred segments, Nguyen et al. proposed in [2] two important properties as follows.

Property 1. Let $M B S_{\nu}(C)$ the sequence of width $\nu$ maximal blurred segments of the curve $C$. Then, $M B S_{\nu}(C)=$ $\left\{M B S\left(B_{1}, E_{1}, \nu\right), M B S\left(B_{2}, E_{2}, \nu\right), \ldots, M B S\left(B_{m}, E_{m}, \nu\right)\right\}$ and satisfies $B_{1}<B_{2}<\ldots<B_{m}$. So we have: $E_{1}<E_{2}<$ $\ldots<E_{m}$.

Property 2. Let $L(k), R(k)$ be the functions which respectively return the index of the left and right extremities of the maximal blurred segments on the left and right sides of the point $C_{k}$. So:

- $\forall k$ such that $E_{i-1}<k \leq E_{i}$, then $L(k)=B_{i}$

- $\forall k$ such that $B_{i} \leq k<B_{i+1}$, then $R(k)=E_{i}$

For a given width $\nu$, the sequence of the maximal blurred segments of a curve $C$ entirely determines the structure of $C$. It can be used for determining the curvature profile which uses Gaussian smoothing [3] or uses osculating circle [2]. Feschet [31] proposed a fast polygon approximation 


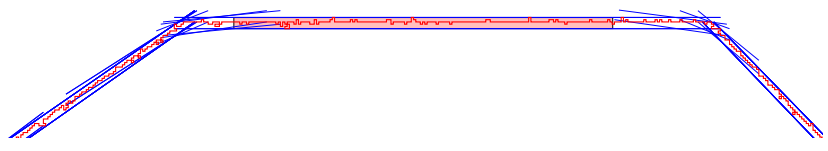

(a) Set of maximal blurred segments on a curve

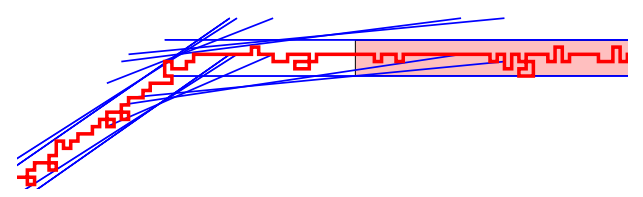

(b) Zoom of (a)

Figure 3: Gray zone is not a common zone of successive maximal blurred segments.

based on the sequence of maximal blurred segments. We propose in the next section a method for dominant point detection constructed from this representation.

\section{Dominant point detection}

Dominant point or corner point is a popular notion in pattern recognition, especially in shape representation. The most critical role of dominant points is that they are partitioning points for the decomposition of a curve into meaningful parts. This is one important step in shape analysis. Many methods have been proposed with several definitions for dominant point detection. We recall here the definition of Attneave in the first work [6] about dominant points.

Definition 6. A dominant point (corner point) on a curve is a point of local maximum curvature.

We can directly detect the dominant points of the curve as the local maximal of its curvature profile [2]. One of the greatest problems is that there can be more weak dominant points that correspond to small local maximal peaks on the curvature profile of the curve. The traditional solutions detect dominant points in multi-scale, multi-resolution by using scale space technique or multi-resolution technique. In this section, we present a new method for dominant point detection based on theoretical results of discrete geometry (recalled in section 2): the sequence of maximal blurred segments of a curve permits to obtain important information about the geometrical structure of the studied curve. The width of the maximal blurred segments, the only parameter in our method, permits to work at different scales and permits to consider the noise which is possibly existing in the curve.

\subsection{Dominant points and region of support (ROS)}

Deducing from [11], we propose the notion of ROS which is compatible with the blurred segment notion.

Definition 7. Width $\nu$ maximal left and right blurred segments of a point constitute its region of support (ROS) (see figure 4). The angle between them is called the ROS angle of this point.

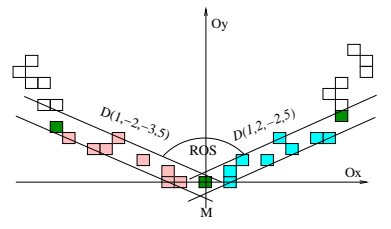

Figure 4: Left, right arm chair and region of support based on left, right extremities of the point $M$.

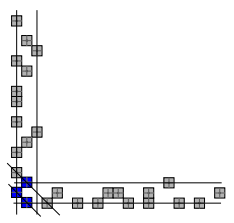

Figure 5: Common zone (in black points) contains a candidate as dominant point
Remark 1. The smaller the ROS angle of a point is, the higher the dominant character of this point is.

This remark is deduced from the work [2], where $c u r$ vature at a point $C_{k}$ is estimated as inverse of the radius of the circumcircle passing through $C_{k}$ and the extremities of its left and right width $\nu$ maximal blurred segments. Therefore, we have a corollary of this remark: if the ROS angle of a point is nearly $180^{\circ}$, this point cannot be a dominant point.

\subsection{Dominant points and maximal blurred segments}

In this section, we study the relation between the dominant points and the sequence of maximal blurred segments. This relation is presented by these propositions below.

Proposition 1. A dominant point of the curve must be in a common zone of successive maximal blurred segments.

Proof 1. Let us consider the points on the pink zone (see figure 3) which are not in a common zone of successive maximal blurred segments but which belong to one blurred segment. By applying property 2, the left and right end points of the blurred segments of these points are also in the same blurred segment. The ROS angles of these points are nearly $180^{\circ}$. Therefore these points are not candidates as dominant points.

Let us now consider the common zone of more than 2 successive maximal blurred segments.

Proposition 2. The smallest common zone of successive width $\nu$ maximal blurred segments, of which slopes are increasing or decreasing, contains a candidate as dominant point (see also figure 5).

Proof 2. Let us consider $k$ successive width $\nu$ maximal blurred segments which share the smallest common zone. Without loss of generality, we assume that these $k$ maximal blurred segments do not intersect any the other smallest zone. Suppose that there are $k$ first maximal blurred segments with the extremities below: $\left(B_{1}, E_{1}\right),\left(B_{2}, E_{2}\right)$, $\ldots,\left(B_{k}, E_{k}\right)$. Their slopes satisfy slope Tl $_{1}<$ slope $_{2}<\ldots<$ slope $_{k}$ (similarity to decreasing case). Due to property 1 , we must have: $B_{1}<B_{2}<\ldots<B_{k} ; E_{1}<E_{2}<\ldots<E_{k}$. Because these maximal blurred segments share the smallest common zone, we must have $B_{k}<E_{1}$. So, the smallest common zone is $\left[B_{k}, E_{1}\right]$. By applying property 2, 


\section{\begin{tabular}{lll}
\hline & B & - \\
\hline
\end{tabular}}

Figure 6: If a maximal blurred segment contains more than 2 dominant point candidates, the middle candidates are weak candidates of dominant point.

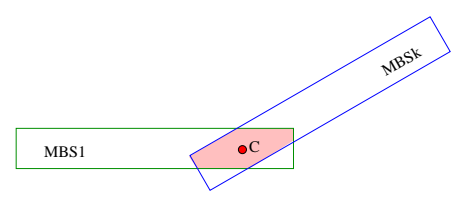

Figure 7: A dominant point is expected as center of the smallest common zone.

the left and right extremities of the points of the $k$ partial common zones $\left[B_{1}, B_{2}\left[,\left[B_{2}, B_{3}\left[, \ldots\left[B_{k}, E_{1}[\right.\right.\right.\right.\right.$ respectively are $\left(B_{1}, E_{1}\right),\left(B_{1}, E_{2}\right), \ldots\left(B_{1}, E_{k}\right)$. The slopes of the left blurred segments of the points of these partial common zones are always equal to slope 1 . On the contrary, the slopes of the right blurred segments of the points of these partial common zones respectively are slope ${ }_{1}$, slope , $_{2}$, ... , slope $_{k}$. By a similar way, we deduce that on the partial common zones $\left.\left.\left.] E_{1}, E_{2}\right], \ldots,\right] E_{k-1}, E_{k}\right]$, the slopes of the right blurred segments of the points of these partial common zones are equal to slope ${ }_{k}$ and the slopes of the left blurred segments respectively are equal to slope $2, \ldots$ , slope $k_{k}$. The ROS angle of the points in the zone $\left[B_{k}, E_{1}\right)$ is equal to the angle $\left(\right.$ slope $_{1}$, slope $\left._{k}\right)$ and this value is minimal for all the points indexed from $B_{1}$ to $E_{k}$, due to the hypothesis of the increasing slopes of maximal blurred segments. Therefore, this zone contains a candidate as dominant point.

To eliminate the weak dominant point candidates, we use the following natural property of a maximal blurred segment, due to the shape of straight line of a maximal blurred segment and also due to the property of corner of a dominant point (see figure 6).

Property 3. A maximal blurred segment contains at most 2 dominant points.

\subsection{Proposed algorithm}

\subsubsection{Algorithm}

We propose below a heuristic strategy for locating the position of each dominant point candidate.

Heuristic strategy: In the each smallest common zone of successive maximal blurred segments of which slopes are increasing or decreasing, a candidate as dominant point is detected as middle point of this zone.

Let us consider the smallest zone that satisfies this condition. This zone contains a candidate as dominant point (cf. proposition 2). By using property 1, this zone must be the intersection of the first and the last maximal blurred segments in the set of successive maximal blurred segments that share this zone (see figure 7 ). We recall that each point in this zone has the same region of support. We then propose to locate the candidate as dominant point which has geometric properties close to the expected corner point. So, the candidate as dominant point is detected as middle point of the partial curve corresponding to this zone.

Based on the above theoretical framework and using the heuristic strategy above, we present hereafter our proposed algorithm for dominant point detection. It is decomposed into two parts :

- the scan of the interesting common zones of maximal blurred segments according to proposition 2 and property 3 ,

- the detection of dominant points in common zones of successive maximal blurred segments of which slopes are increasing or decreasing.

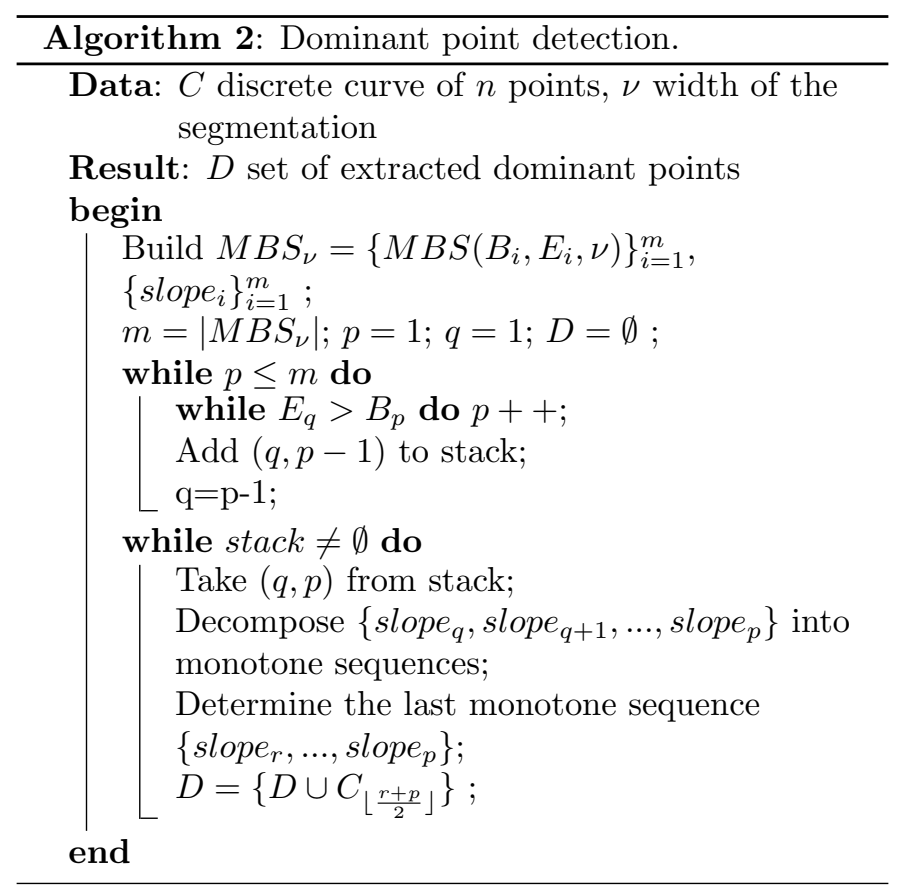

\subsubsection{Complexity}

The complexity of our method depends on the decomposition of a curve into maximal blurred segments. We can use our previous technique [2] that can be done in $O\left(n \log ^{2} n\right)$ time. Recently, Faure [5] has proposed a technique for tangential cover that corresponds exactly to this problem of decomposition. The complexity of this technique is in $O(n \log n)$. The slope estimation of maximal blurred segments is done in linear time. On the other hand, each maximal blurred segment is considered at most twice while the curve is decomposed into common zone of maximal blurred segments of which slopes are monotone sequence. So, in this phase, the dominant points are detected in linear time. Therefore the complexity of this proposed method is in $O(n \log n)$ thanks to Faure's improvement. 


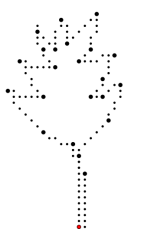

(a) Leaf

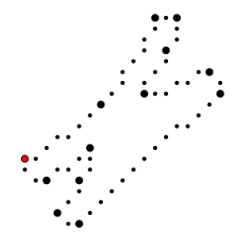

(b) Chromosome

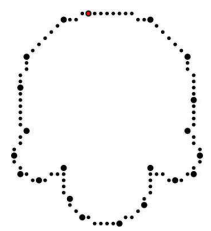

(c) Semicircle
Figure 8: Detected dominant points at width parameter 0.9.

\section{Experimentation}

\subsection{Experimentation and comparison with other methods}

We present in figure 8 our obtained results on 3 wellknown curves: chromosome, leaf, semicircle. Figure 9 shows some experimentation of the proposed method on simple shapes in comparison with Marji's method. Figures 10, 11, 12 and 13 show the comparison with the results obtained by other methods on 3 well-known curves: chromosome, leaf and semicircle.

We have compared our method with others (see table 3) on some criteria: number of dominant points (nDP), compression ratio (CR), ISE error, max error, and figure of merit (FOM). CR is the ratio between number of curve points and number of detected dominant points.

$$
C R=\frac{n}{n D P}
$$

ISE is sum of squared perpendicular distance of the curve points from approximating polygon.

$$
I S E=\sum_{i=1}^{n} d_{i}^{2}
$$

Max error is the highest perpendicular distance of the curve points from approximating polygon.

$$
L_{\infty}=\max \left\{d_{i}\right\}_{i=1}^{n}
$$

where $d_{i}$ is perpendicular distance from $i^{t h}$ curve point to approximating polygon. Because a low error approximation leads to a low compression ratio, Sarkar[13] propose FOM criterion to combine these measures. FOM is estimated as ratio between $\mathrm{CR}$ and ISE.

$$
F O M=\frac{C R}{I S E}
$$

Because FOM criterion is not suitable for comparison with different dominant point number, Rosin [27] proposed other criteria to evaluate obtained result by comparing it with optimal result. He splitted the assessment into two measures: fidelity and efficiency. Fidelity measures how well the sub-optimal polygon fits the curve relative to the optimal polygon in term of approximation error.

$$
\text { Fidelity }=\frac{E_{\text {opt }}}{E_{\text {appr }}} * 100
$$

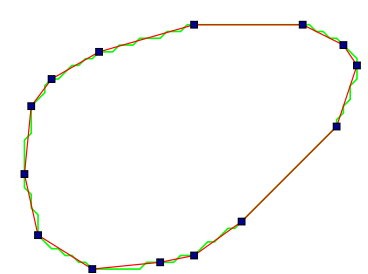

(a) Proposed

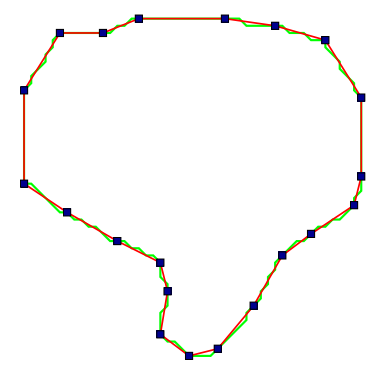

(c) Proposed

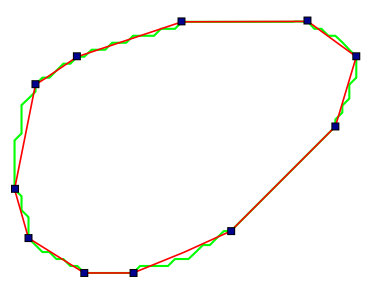

(b) Marji

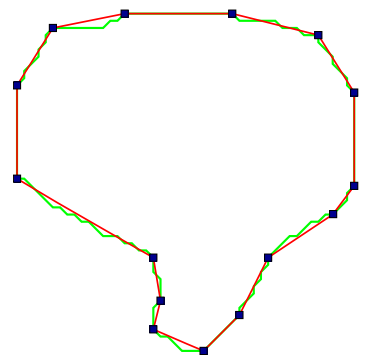

(d) Marji
Figure 9: Comparison between the proposed method at default parameter and Marji's one [32] on 2 simple shapes (see also table 1).

\begin{tabular}{|c|c|c|c|c|c|c|}
\hline Curve & $N_{P}^{0}$ & Method & $N_{D P}^{0}$ & CR & ISE & FOM \\
\hline \multirow{2}{*}{ (a)-(b) } & \multirow{2}{*}{123} & Marji & 11 & 11.182 & 24.67 & 0.453 \\
\cline { 3 - 7 } & & Ours & 14 & 8.786 & 9.296 & 0.945 \\
\hline \multirow{2}{*}{ (c)-(d) } & \multirow{2}{*}{142} & Marji & 15 & 9.467 & 21.508 & 0.44 \\
\cline { 3 - 7 } & Ours & 21 & 6.762 & 8.782 & 0.77 \\
\hline
\end{tabular}

Table 1: Comparison with Marji's method on figure 9 by using Sarkar's criterion $\left(N_{P}^{0}\right.$ number of points, $N_{D P^{-}}^{0}$ number of dominant points, CR- compression rate, ISE- integrated square error, FOM- figure of merit). 


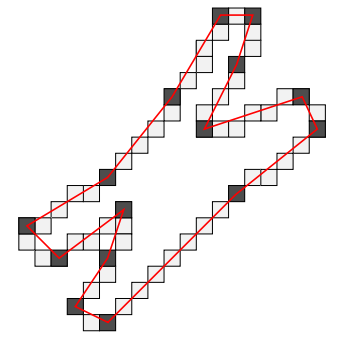

(a)

Proposed

width $=0.8$

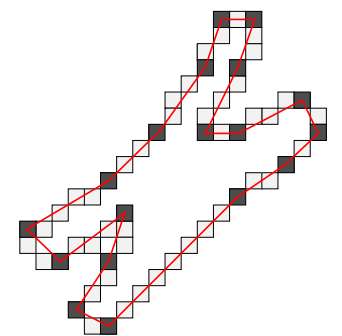

(f)

width $=0.7$

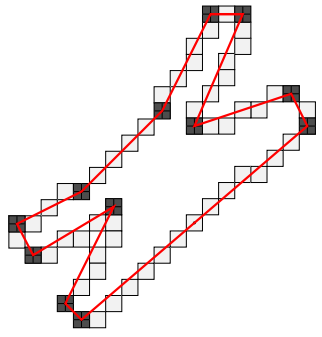

(b) Marji [12]

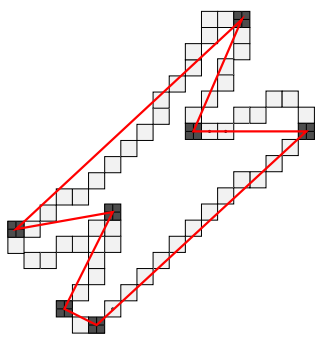

(g) Arcelli [34]

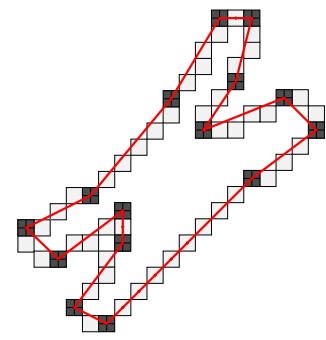

(c) $\operatorname{Teh}[11]$

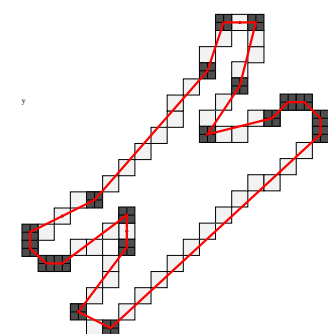

(h) Sarkar [13]

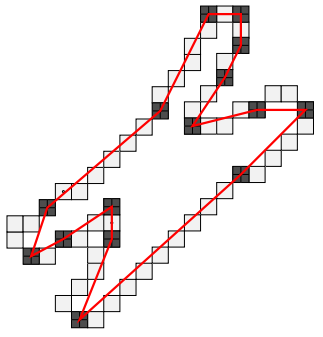

(d) Ansari [33]

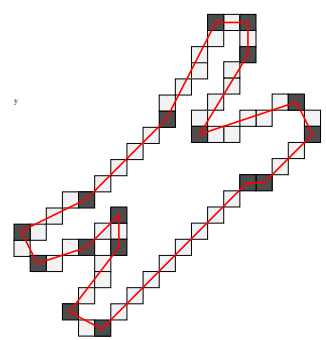

(i) Cronin [14]

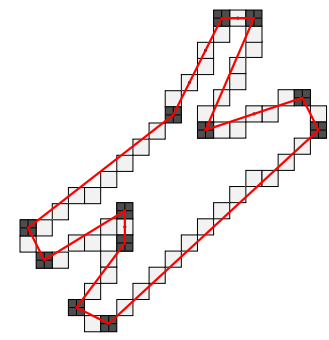

(e) Masood[25]

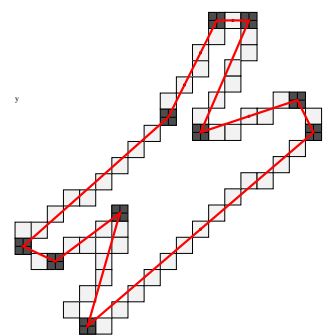

(j) Shearer [35]

Figure 10: Dominant points of the chromosome curve.

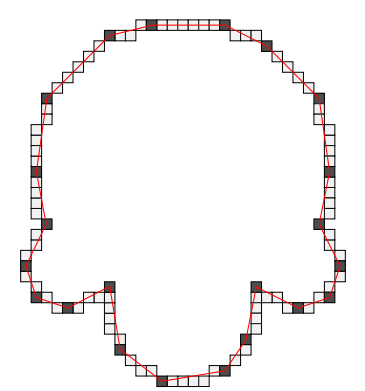

(a) Proposed, width $=0.8$

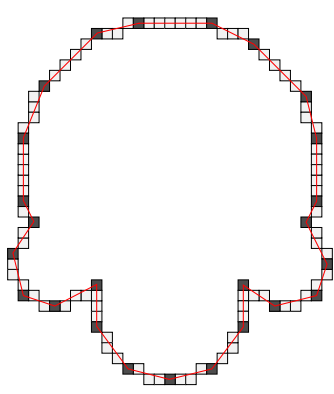

(f) Proposed, width $=0.7$

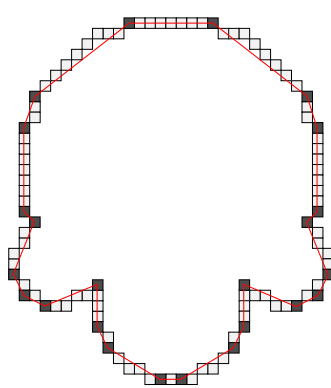

(b) Marji [32]

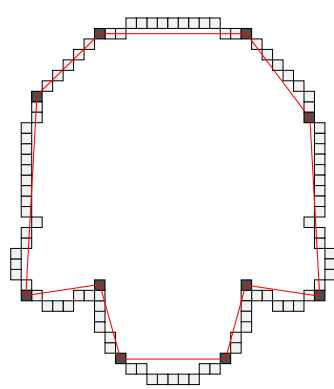

(g) Arcelli [34]

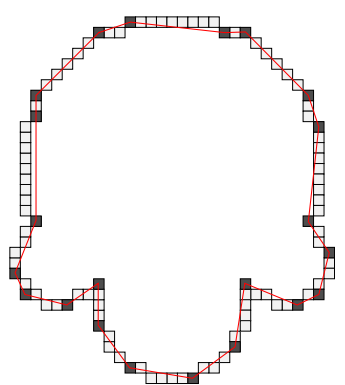

(c) $\operatorname{Teh}[11]$

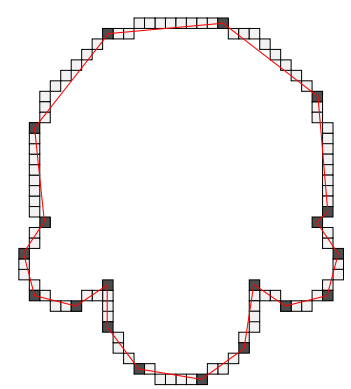

(h) Sarkar [13]

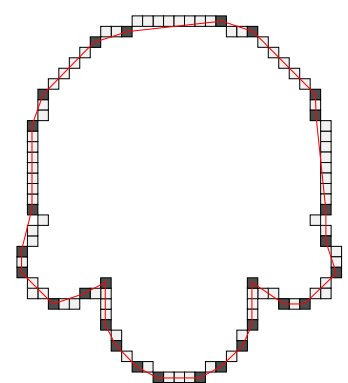

(d) Ansari [33]

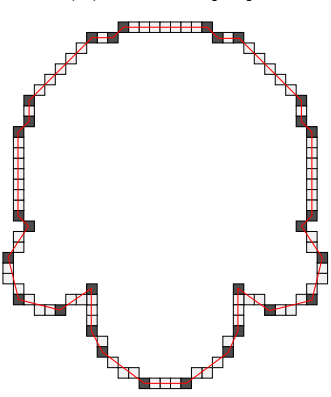

(i) Cronin [14]

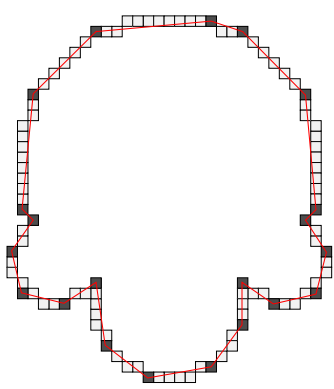

(e) Masood[25]

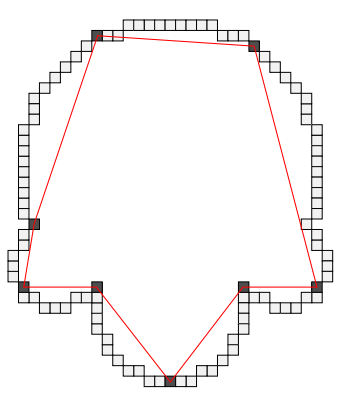

(j) Quddus [36]

Figure 11: Dominant points of the semicircle curve. 


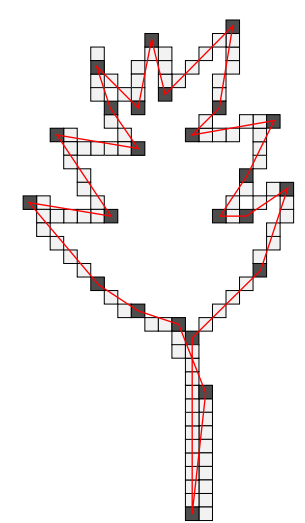

(a)

width $=0.8$

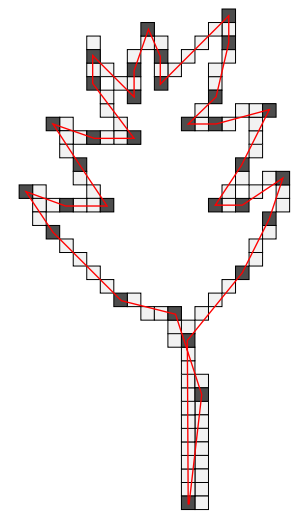

(f) width $=0.7$

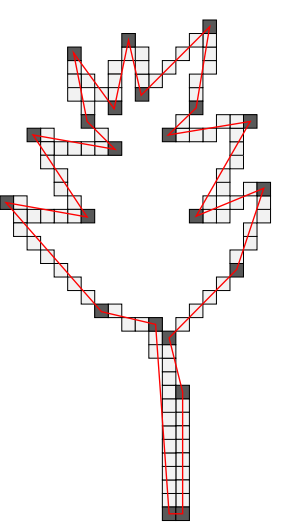

(b) Marji [32]

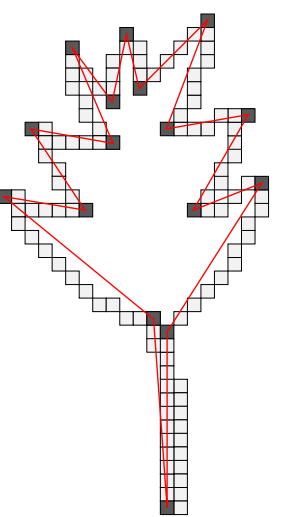

(g) Arcelli[34]

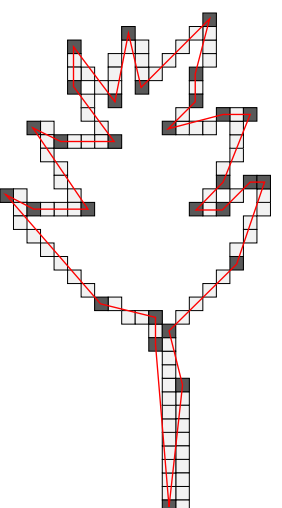

(c) Teh-Chin[11]

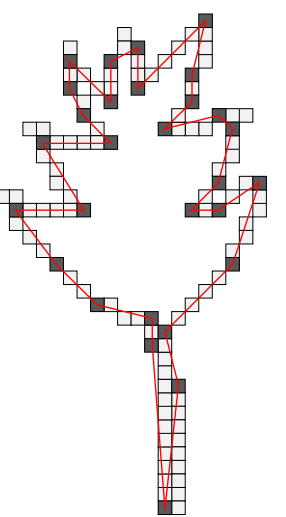

(d) Ansari [33]

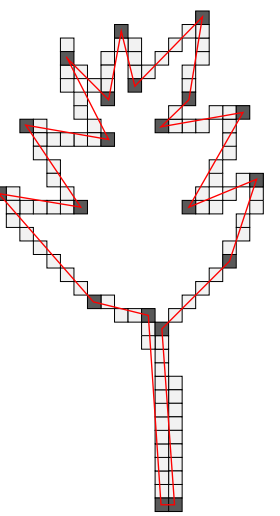

(e) Masood[25]
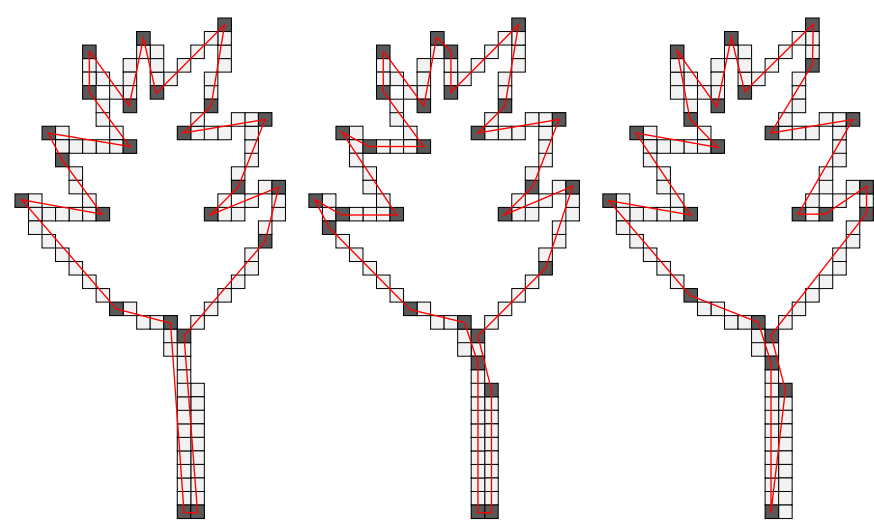

(h) Sarkar [13]

(i) Cronin $[14]$

(j) Poyato [37]

Figure 12: Dominant points of the leaf curve. 


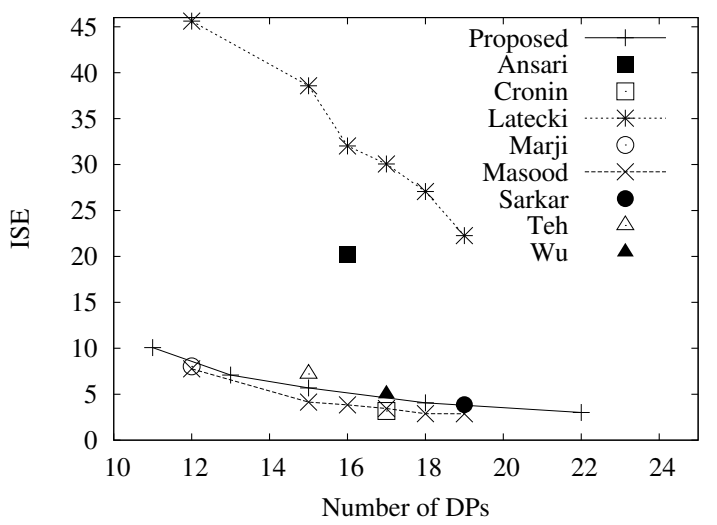

(a) Chromosome curve

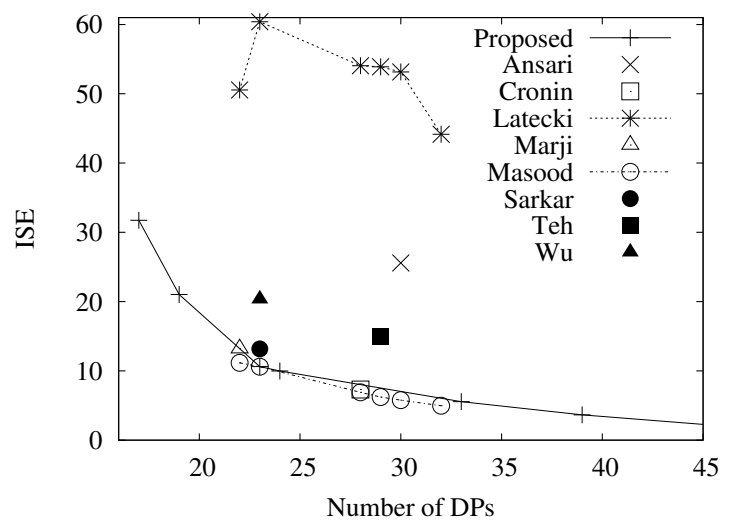

(b) Leaf curve

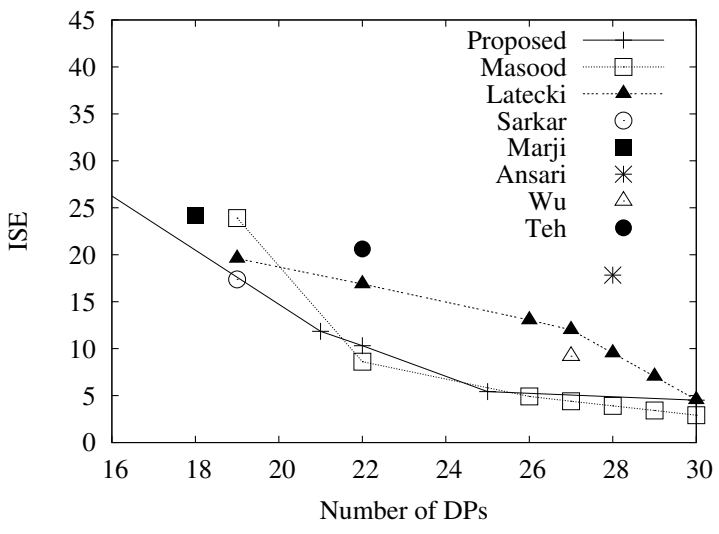

(c) Semicircle curve

Figure 13: Relation between the number of dominant points and the ISE among different methods: our method, Massood [25], Latecki [26], Shearer [35], Wu [38], Marji [12], Teh [11], Ansari[33], Sarkar [13], Cronin [14] on 3 curves (chromosome, leaf and semicircle). 
Efficiency measures how compact the sub-optimal polygon is according to the optimal polygon with the same error.

$$
\text { Efficiency }=\frac{N_{\text {opt }}}{N_{\text {appr }}} * 100
$$

where $E_{a p p r}$ and $N_{a p p r}$ is error and DP number of tested algorithm, $E_{\text {opt }}$ is error of optimal algorithm with the same approximated DP number, $N_{o p t}$ is DP number of optimal algorithm with the same approximated error. The merit measure is based on these measures.

$$
\text { Merit }=\sqrt{\text { Fidelity } * \text { Efficency }}
$$

Tables 1, 3 show a comparison between our method and other methods by using Sarkar's criterion. In addition, the table 4 gives a comparison based on Rosin's point of view. Our method is better than 28 methods among 30 ones given in Rosin's list [27] (see table 4).

On the other hand, by changing the value of the width parameter, we obtain different numbers of dominant points with corresponding error approximation for each curve. Figure 13 shows the relation between the number of dominant points and the ISE among different methods when they are applied for 3 curves: leaf, chromosome and semicircle. Thanks to this graph, we recognize that our method is a little less efficient than Masood's method but it is more efficient than most of other methods. For example, let us see the case of semicircle curve. For 22 dominant points, ISE value of the proposed method is more lower than Latecki [26] and Teh [11], and is little bigger than Masood [25]. For 21 and 25 dominant points, the proposed method seems to be better than the results of Masood (see figure 13.c).

\subsection{Working with possibly noisy or disconnected curves}

By using the notion of blurred segment, our method can work with possibly noisy or disconnected curves. On the other hand, the width parameter allows us to work with a curve at different scales. Figure 14 presents an experimentation on noisy curves which are generated by using Kanungo model [39]. In addition, figure 15 shows that the proposed method can work well with disconnected curves.

An interesting problem is raised: For each noisy curve, can we determined the best width parameter? We propose to consider a multi-width framework by using an evaluation criterion to choose the best width parameter. In an analysis with multi-scale approach, the simple Sarkar's model seems to get better results than that Rosin's model thanks to its linear complexity in relation with the application of optimal polygonal approximation methods. Therefore, we use Sarkar's criterion to determine the best width parameter through multi-scale framework that is illustrated in algorithm 3. In practice, we choose $\max W i d t h$ as 20 to consider the multi-width process, so the complexity of algorithm 3 is also $O(n \log n)$.

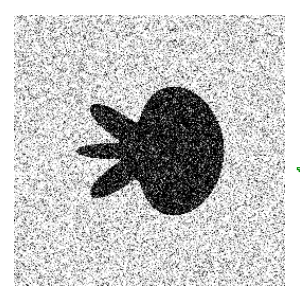

(a) Degradation

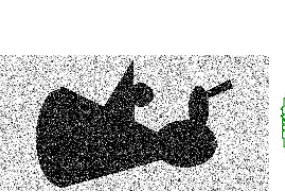

(d) Degradation

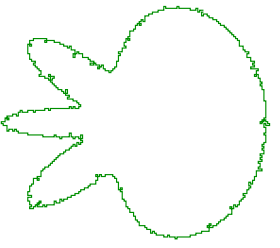

(b) Contour

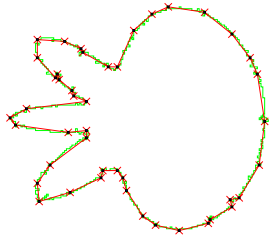

(c) Result

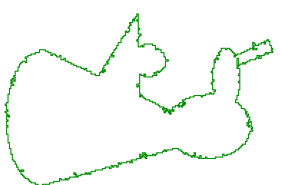

(e) Contour

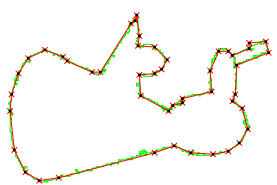

(f) Result
Figure 14: Experimentation of the proposed method on noisy curves by using Kanungo model [39]. The working width is 2 .

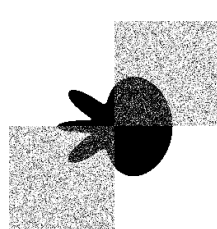

(a) Degraded (b) image

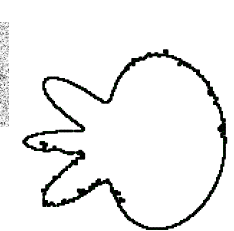

Disconnected contour

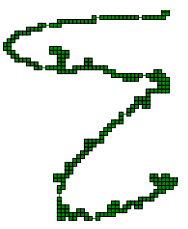

(b)

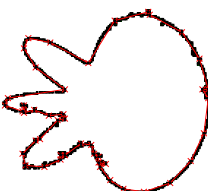

(d) Result
Figure 15: Experimentation on a disconnected noisy curve. The working width is 2 .

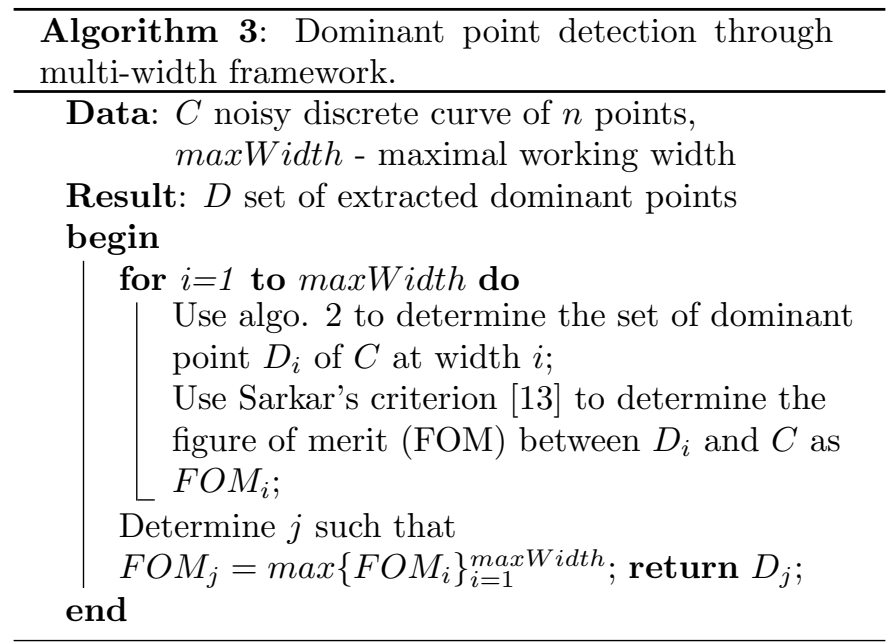



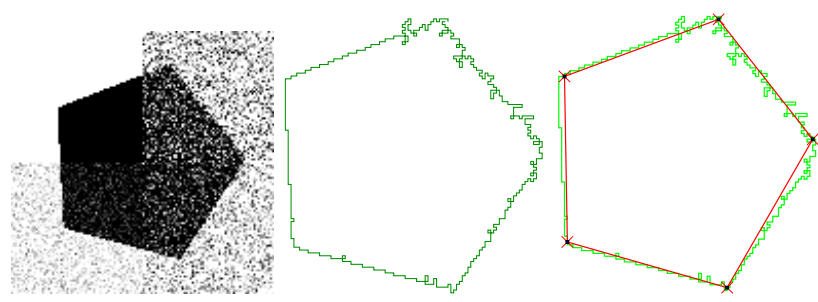

(a) Degraded image tou

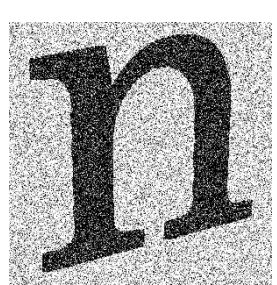
tour

(c) Result, optimal

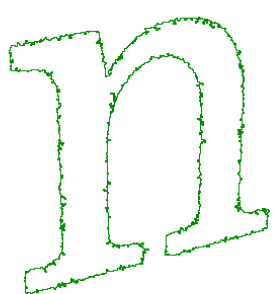

width $=9$

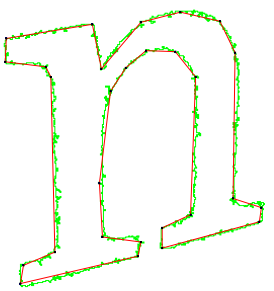

(d) Degraded image (e)

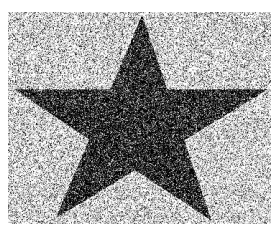

(f) Result, optimal
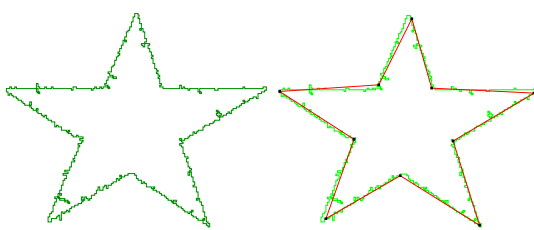

(g) Degraded image (h) Extracted con- (i) Result, optimal tour width $=6$

Figure 16: Corner detection through multi-width framework (see also figure 18.a).

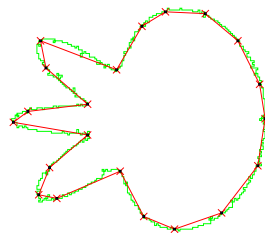

(a) Figure 14.c Optimal width: 5

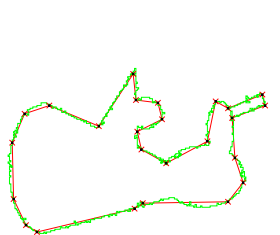

(b) Figure 14.g Optimal width: 4

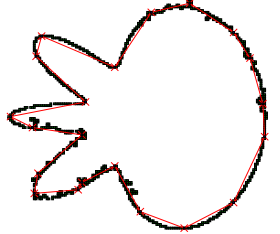

(c) Figure 15.b Optimal width: 5
Figure 17: Dominant point detection results based on algorithm 3 on the curves in figures 14 and 15 (see also figure 18.b).

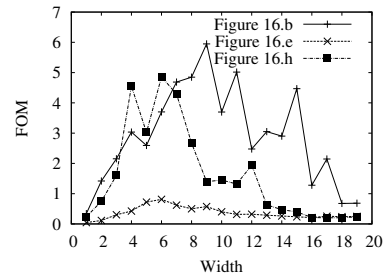

(a) FOM profile in figure 16

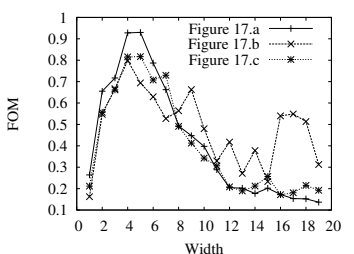

(b) FOM profile in figure 17
Figure 18: Sarkar's FOM profile through multi-width process.

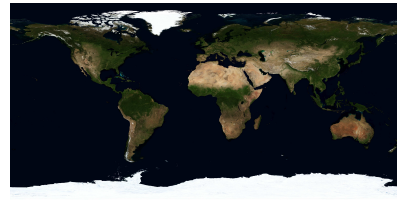

(a) 5400x2700 pixels

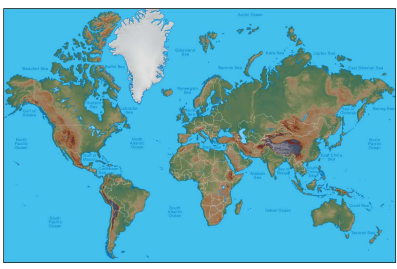

(b) 1200x778 pixels

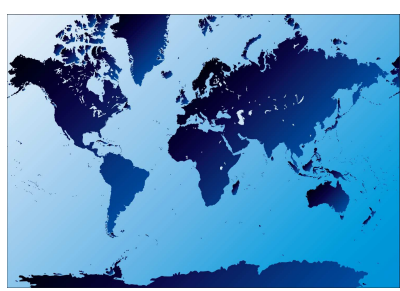

(d) 1500x1043 pixel (c) 2000x1000 pixels

Figure 19: World map dataset.

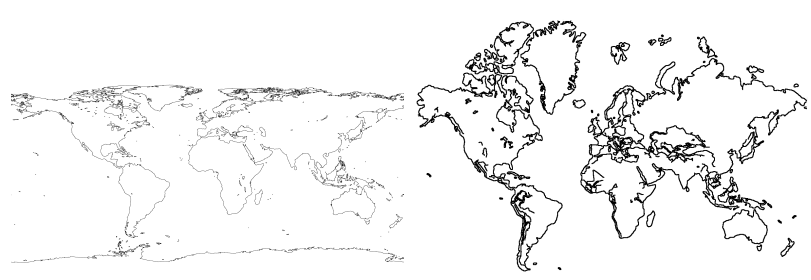

(a) 1781 curves of 103319 (b) 240 curves of 18371 points points
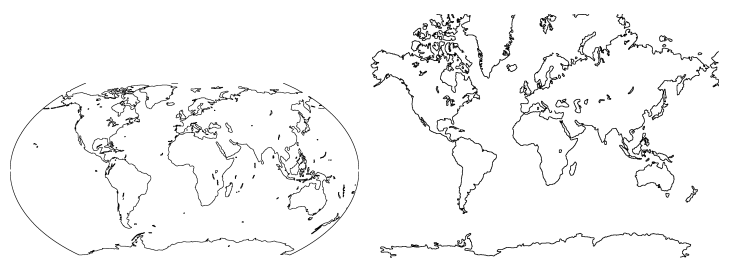

(c) 334 curves of 25105 (d) 170 curves of 20781 points points

Figure 20: Edges of world map on figure 19. ${ }^{a}$

${ }^{a}$ For the best display, the edge images are eroded in this figure.

Figure 16 presents an example with noisy curves extracted from degraded images. Figure 17 presents the results of dominant point detection on the curves in figures 14.c, .g and 15.b based on algorithm 3. These results are obtained at the best corresponding width parameters through a multi-width framework vs. a fixed parameter in figures 14 and 15 .

\section{Application to vectorization}

We apply the proposed method for an application of vectorization. Figure 19 presents a small dataset of 4 different images whose edge images are given in figure 20 by using Canny filter. Each edge image is considered as a set of contour curves. The proposed method is applied on these contour curves to obtain the corresponding image of vectorization. 


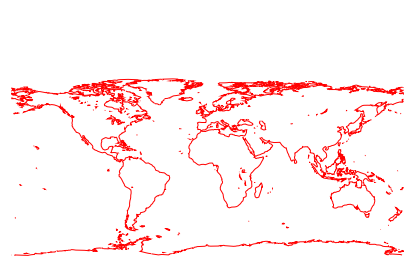

(a) Figure 20.a

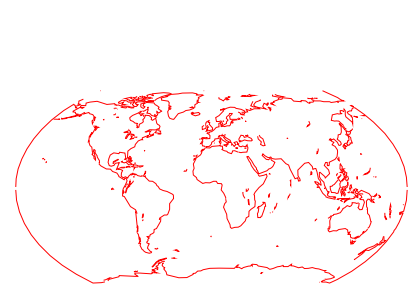

(c) Figure 20.c

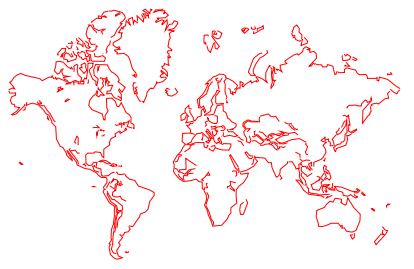

(b) Figure 20.b

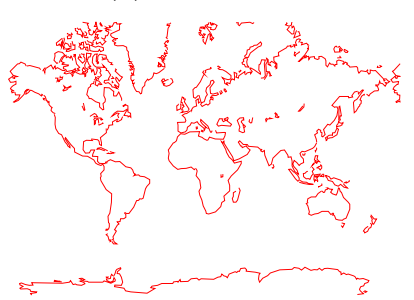

(d) Figure 20.d
Figure 21: Vectorization of world map dataset in figure 20. The working width is 2 .

\begin{tabular}{|c|c|c|c|c|c|}
\hline Fig. & $N_{C}^{0}$ & $N_{P}^{0}$ & $N_{D P}^{0}$ & CR & Time \\
\hline 21.a & 1781 & 103319 & 14473 & 7.139 & 6019 \\
\hline 21.b & 240 & 18371 & 1818 & 10.1 & 1071 \\
\hline 21.c & 334 & 25105 & 2094 & 11.989 & 1671 \\
\hline 21.d & 170 & 20781 & 1949 & 10.662 & 1367 \\
\hline
\end{tabular}

Table 2: Vectorization results on figure 21. Time is computed in ms, $N_{C}^{0}$ - number of curves, $N_{P}^{0}$ - number of points, $N_{D P}^{0}$ - number of dominant point, $\mathrm{CR}$ - compression rate.

Figure 21 and table 2 show the obtained results by the proposed method on these edge images. The experimentations are undertaken on a configuration of computer as follows: CPU Pentium 4 with $2.26 \mathrm{GHz}, 4 \mathrm{G}$ of RAM, linux kernel 2.6.31-20 operating system. The proposed method can work with $10^{5}$ points in 6 seconds (see table 2 ).

\section{Conclusion}

Detection of dominant points on the curves is a traditional topic in shape representation. It implies applications in image analysis, vectorization and shape matching. In this paper, we have presented a new method for dominant point detection based on the point of view of the discrete geometry. This method utilizes the recent results in this domain to work naturally with noisy curves. The result of this method has been compared with many other methods. It shows its ability to produce high quality approximations with default parameters. By using width parameter, this method can also work at different scales.

We also investigate this method for shape matching through multi-width approach. By using Sarkar's evaluation criterion (FOM - figure of merit), we can determine the best value of width parameter by regarding the profile of FOM through the multi-width process. The best value is chosen as the highest value on this profile. This simple idea gives good results on noisy curves. In addition, it uses no threshold to work with noisy curves.
The proposed methods have a low complexity $(O(n \log n))$ and they can work with noisy curves and possibly disconnected curves. Therefore, they lead to real applications in machine vision, data compression, vectorization. . . An application of vectorization has been proposed in this work. In the future, we are interested in other applications.

\section{Acknowledgment}

We would like to thank anonymous reviewers for their valuable comments to improve this work.

\section{References}

[1] T. P. Nguyen, I. Debled-Rennesson, Fast and robust dominant point detection on digital curves, in: ICIP, 2009, pp. 953-956.

[2] T. P. Nguyen, I. Debled-Rennesson, Curvature estimation in noisy curves, in: CAIP, Vol. 4673 of LNCS, 2007, pp. 474-481.

[3] F. Feschet, L. Tougne, Optimal time computation of the tangent of a discrete curve: Application to the curvature., in: DGCI, Vol. 1568 of LNCS, 1999, pp. 31-40.

[4] I. Debled-Rennesson, F. Feschet, J. Rouyer-Degli, Optimal blurred segments decomposition of noisy shapes in linear time, Computers \& Graphics 30 (1) (2006) 30-36.

[5] A. Faure, F. Feschet, Tangential cover for thick digital curves, in: DGCI, Vol. 4992 of LNCS, 2008, pp. 358-369.

[6] E. Attneave, Some informational aspects of visual perception, Psychol. Rev. 61 (3) (1954) 183-193.

[7] F. Zhao, Q. Huang, W. Gao, Image matching by multiscale oriented corner correlation, in: ACCV (1), Vol. 3851 of LNCS, 2006, pp. 928-937.

[8] A. Kolesnikov, Optimal algorithm for lossy vector data compression, in: ICIAR, Vol. 4633 of LNCS, 2007, pp. 761-771.

[9] B. Kerautret, J.-O. Lachaud, B. Naegel, Comparison of discrete curvature estimators and application to corner detection, in: ISVC (1), Vol. 5358 of LNCS, 2008, pp. 710-719.

[10] A. Rosenfeld, E. Johnston, Angle detection on digital curves, IEEE Trans. Comput. 22 (1973) 940-941.

[11] C.H.Teh, R.T.Chin, On the detection of dominant points on the digital curves, IEEE Trans. Pattern Anal. Mach. Intell. 2 (1989) 859-872

[12] M. Marji, P. Siy, A new algorithm for dominant points detection and polygonization of digital curves, Pattern Recognition 36 (10) (2003) 2239-2251.

[13] D. Sarkar, A simple algorithm for detection of significant vertices for polygonal approximation of chain-coded curves, Pattern Recognition Letters 14 (12) (1993) 959-964.

[14] T. M. Cronin, A boundary concavity code to support dominant point detection, Pattern Recognition Letters 20 (6) (1999) 617634.

[15] H. Freeman, On the encoding of arbitrary geometric configurations, IRE Trans. Electron. Comput. 10 (1961) 260-268.

[16] B. K. Ray, K. S. Ray, Determination of optimal polygon from digital curve using $L_{1}$ norm, Pattern Recognition 26 (4) (1993) 505-509.

[17] A. Kolesnikov, P. Fränti, Polygonal approximation of closed discrete curves, Pattern Recognition 40 (4) (2007) 1282-1293.

[18] H. Aoyama, M. Kawagoe, A piecewise linear approximation method preserving visual feature points of original figures, CVGIP: Graph. Models Image Process. 53 (5) (1991) 435-446.

[19] A. Held, K. Abe, C. Arcelli, Towards a hierarchical contour description via dominant point detection, Systems, Man and Cybernetics, IEEE Transactions on 24 (6) (1994) 942-949.

[20] M. Sarfraz, M. Asim, A. Masood, Piecewise polygonal approximation of digital curves, Information Visualisation, 2004. IV 2004. Proceedings. Eighth International Conference on (2004) 991-996. 


\begin{tabular}{|c|c|c|c|c|c|c|}
\hline Shape & Algorithm & $n D P$ & $C R$ & $L_{\infty}$ & $I S E$ & $F O M$ \\
\hline \multirow{10}{*}{ Chromosome } & Proposed, width $=0.7$ & 18 & 3.3333 & 0.6325 & 4.0596 & 0.8211 \\
\hline & Sharkar [13] & 19 & 3.16 & 0.55 & 3.857 & 0.819 \\
\hline & Shearer [35] & 10 & 6 & & 6.086 & 0.744 \\
\hline & $\mathrm{Wu}[38]$ & 17 & 3.53 & 0.64 & 5.01 & 0.704 \\
\hline & Proposed, width $=0.8$ & 15 & 4 & 0.686 & 5.691 & 0.7028 \\
\hline & Ray[40] & 18 & 3.33 & 0.65 & 4.81 & 0.693 \\
\hline & Masood [25] & 12 & 5 & 0.88 & 7.76 & 0.65 \\
\hline & Ray [41] & 18 & 3.33 & 0.707 & 5.566 & 0.599 \\
\hline & Teh [11] & 15 & 4.00 & 0.74 & 7.2 & 0.556 \\
\hline & Marji [12] & 11 & 5.45 & 0.895 & 9.96 & 0.548 \\
\hline \multirow{10}{*}{ Leaf } & Proposed, width $=0.7$ & 33 & 3.636 & 0.632 & 5.5604 & 0.654 \\
\hline & Proposed, width $=0.8$ & 24 & 5 & 0.7276 & 9.9883 & 0.5006 \\
\hline & Masood [25] & 23 & 5.217 & 0.74 & 10.61 & 0.49 \\
\hline & Shearer [35] & 22 & 5.46 & & 13.06 & 0.418 \\
\hline & Marji [12] & 22 & 5.45 & 0.78 & 13.21 & 0.413 \\
\hline & Sarkar [13] & 23 & 5.22 & 0.784 & 13.17 & 0.396 \\
\hline & Teh [11] & 29 & 4.14 & 0.99 & 14.96 & 0.277 \\
\hline & Ray [40] & 32 & 3.75 & 0.99 & 14.18 & 0.264 \\
\hline & Ray [41] & 32 & 3.75 & 0.996 & 14.718 & 0.255 \\
\hline & $\mathrm{Wu}[38]$ & 23 & 5.22 & 1 & 20.34 & 0.256 \\
\hline \multirow{11}{*}{ Semicircle } & Proposed (width $=0.7$ ) & 25 & 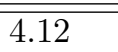 & 0.5571 & 5.4226 & 0.76 \\
\hline & Masood [25] & 22 & 4.64 & 0.72 & 8.61 & 0.54 \\
\hline & Proposed $($ width $=0.8)$ & 22 & 4.681 & 0.784 & 10.31 & 0.454 \\
\hline & $\mathrm{Wu}[38]$ & 27 & 3.78 & 0.88 & 9.19 & 0.411 \\
\hline & Ray [40] & 27 & 3.78 & 0.88 & 11.5 & 0.329 \\
\hline & Sarkar [13] & 19 & 5.37 & 1.474 & 17.37 & 0.309 \\
\hline & Ray $[41]$ & 29 & 3.52 & 0.833 & 11.818 & 0.298 \\
\hline & Ansari-Huang [33] & 28 & 3.64 & 1.26 & 17.83 & 0.24 \\
\hline & Marji [12] & 18 & 5.67 & 1 & 24.2 & 0.234 \\
\hline & Masood [25] & 19 & 5.37 & 1.00 & 23.9 & 0.23 \\
\hline & Teh [11] & 22 & 4.64 & 1 & 20.61 & 0.225 \\
\hline
\end{tabular}

Table 3: Comparison using Sarkar's criterion [13].

\begin{tabular}{|l|l|l|l|l|l|l|}
\hline Method & nDP & ISE & Fidelity & Efficiency & Merit & $\begin{array}{l}\text { Rosin's } \\
\text { rank }\end{array}$ \\
\hline Proposed (width=0.7) & 25 & 5.422 & 86.4 & 96 & 91.08 & - \\
\hline Massod [25] & 21 & 9.82 & 81.7 & 95.8 & 88.5 & - \\
\hline Ramer [42] & 26 & 5.27 & 76.9 & 92.6 & 84.4 & 2 \\
\hline Proposed (width=0.8) & 22 & 10.31 & 67.99 & 86.36 & 76.6 & - \\
\hline Sarkar II[13] & 20 & 13.65 & 66 & 78.9 & 72.2 & 4 \\
\hline Marji [32] & 15 & 22.7 & 61.67 & 84.0 & 71.9 & - \\
\hline Sarkar I[13] & 19 & 17.38 & 57.8 & 73.7 & 65.3 & 6 \\
\hline Arcelli-Ramella [34] & 10 & 75.10 & 51.8 & 80.3 & 64.5 & 7 \\
\hline Wu [38] & 27 & 9.01 & 41.09 & 74.07 & 55.17 & - \\
\hline Teh [11] & 22 & 20.61 & 34.0 & 59.2 & 42.4 & 17 \\
\hline Ansari-Huang [33] & 28 & 17.83 & 18.8 & 49.1 & 28.8 & 26 \\
\hline Ray [41] & 29 & 11.82 & 25.4 & 60.0 & 39.0 & 23 \\
\hline
\end{tabular}

Table 4: Comparison using Rosin's criterion [27] on the semicircle curve. 
[21] S.-C. Huang, Y.-N. Sun, Polygonal approximation using genetic algorithms, Pattern Recognition 32 (8) (1999) 1409-1420.

[22] H. Zhang, J. Guo, Optimal polygonal approximation of digital planar curves using meta heuristics, Pattern Recognition 34 (7) (2001) 1429-1436.

[23] P.-Y. Yin, Ant colony search algorithms for optimal polygonal approximation of plane curves, Pattern Recognition 36 (8) (2003) 1783-1797.

[24] L. Li, W. Chen, Corner detection and interpretation on planar curves using fuzzy reasoning, IEEE Transactions on Pattern Analysis and Machine Intelligence 21 (11) (1999) 1204-1210.

[25] A. Masood, Dominant point detection by reverse polygonization of digital curves, Image Vision Comput. 26 (5) (2008) 702-715.

[26] L. J. Latecki, R. Lakämper, Polygon evolution by vertex deletion, in: Scale-Space, Vol. 1682 of LNCS, 1999, pp. 398-409.

[27] P. L. Rosin, Techniques for assessing polygonal approximations of curves, IEEE Trans. Pattern Anal. Mach. Intell. 19 (6) (1997) 659-666.

[28] J.-P. Reveillès, Géométrie discrète, calculs en nombre entiers et algorithmique, thèse d'état. Université Louis Pasteur, Strasbourg (1991).

[29] M. Overmars, J. van Leeuwen, Maintenance of configurations in the plane, J. Comput. and Syst. Sci. 23 (1981) 166-204.

[30] L. Buzer, An elementary algorithm for digital line recognition in the general case., in: DGCI, Vol. 3429 of LNCS, 2005, pp. 299-310.

[31] F. Feschet, Fast guaranteed polygonal approximations of closed digital curves, in: SCIA, Vol. 3540 of LNCS, 2005, pp. 910-919.

[32] M. Marji, P. Siy, Polygonal representation of digital planar curves through dominant point detection - a nonparametric algorithm, Pattern Recognition 37 (11) (2004) 2113-2130.

[33] N. Ansari, K. wei Huang, Non-parametric dominant point detection, Pattern Recognition 24 (9) (1991) 849-862.

[34] C. Arcelli, G. Ramella, Finding contour-based abstractions of planar patterns, Pattern Recognition 26 (10) (1993) 1563-1577.

[35] M. Shearer, J. J. Zou, Detection of dominant points based on noise suppression and error minimisation, in: ICITA '05, Vol. 2, IEEE Computer Society, 2005, pp. 772-775.

[36] A. Quddus, M. Gabbouj, Wavelet-based corner detection technique using optimal scale, Pattern Recognition Letters 23 (1-3) (2002) 215-220.

[37] Á. C. Poyato, N. L. F. García, R. M. Carnicer, F. J. MadridCuevas, Dominant point detection: A new proposal, Image Vision Comput. 23 (13) (2005) 1226-1236.

[38] W.-Y. Wu, An adaptive method for detecting dominant points, Pattern Recognition 36 (10) (2003) 2231-2237.

[39] T. Kanungo, R. M. Haralick, H. S. Baird, W. Stuetzle, D. Madigan, Document degradation models: Parameter estimation and model validation, in: MVA, 1994, pp. 552-557.

[40] B. K. Ray, K. S. Ray, An algorithm for polygonal approximation of digitized curves, Pattern Recognition Letters 13 (7) (1992) 489-496.

[41] B. K. Ray, K. S. Ray, Detection of significant points and polygonal approximation of digitized curves, Pattern Recognition Letters 13 (6) (1992) 443-452.

[42] U.Ramer, An iterative procedure for the polygonal approximation of planar curves, Computer, Graphic and Image processing 1 (1972) 224-256. 